\title{
Electrochemical Monitoring of ROS/RNS Homeostasis Within Individual Phagolysosomes Inside Single Macrophages
}

\author{
Xin-Wei Zhang, ${ }^{1}$ Alexander Oleinick, ${ }^{2}$ Hong Jiang, ${ }^{1}$ Quan-Lan Liao, ${ }^{1}$ Quan-Fa Qiu, ${ }^{1}$ Irina \\ Svir, ${ }^{2}$ Yan-Ling Liu, ${ }^{1}$ Christian Amatore, ${ }^{2,3 *}$ and Wei-Hua Huang ${ }^{1 *}$ \\ ${ }^{1}$ Key Laboratory of Analytical Chemistry for Biology and Medicine (Ministry of Education), College of Chemistry and Molecular \\ Sciences, Wuhan University, Wuhan 430072 (China) \\ 2 PASTEUR, Départment de Chimie, École Normale Supérieure, PSL, Research University, Sorbonne Universités, UPMC Univ. \\ Paris 06, CNRS \\ ${ }^{3}$ State Key Laboratory of Physical Chemistry of Solid Surfaces, College of Chemistry and Chemical Engineering, Xiamen \\ University, Xiamen, 361005 (China) \\ E-mail: whhuang@whu.edu.cn, christian.amatore@ens.fr
}

\begin{abstract}
Reactive Oxygen/Nitrogen Species (ROS/RNS) produced by macrophages inside their phagolysosomes are closely related to immunity and inflammation by being involved in the removal of pathogens, altered cells, etc. The existence of a homeostatic mechanism regulating the ROS/RNS amounts inside phagolysosomes has been invoked to account for the efficiency of this crucial process but this could never be unambiguously documented. In this work, intracellular electrochemical analysis with platinized nanowires electrodes (Pt-NWEs) allowed monitoring ROS/RNS effluxes with sub-millisecond resolution from individual phagolysosomes randomly impacting onto the electrode inserted inside a living macrophage. This evidenced for the first time that the consumption of ROS/RNS by their oxidation at the nanoelectrode surface stimulates the production of significant ROS/RNS amounts inside phagolysosomes. These results established the existence of the long-time postulated ROS/RNS homeostasis and allowed quantifying its kinetics and efficiency. ROS/RNS concentrations may then be maintained at sufficiently high levels for sustaining proper pathogen digestion rates without endangering the macrophage internal structures.
\end{abstract}

Macrophages are essential in protecting our body against bacteria, viruses and mutant or abnormal cells. ${ }^{[1]}$ They detect and internalize such pathogens within endocytotic vesicles named phagosomes. These are carried inside the macrophages cytoplasm where they merge with lysosomes, another kind of vesicle, whose membranes host a panoply of enzymes including NADPH oxidase 2 (NOX2) and inducible nitric oxide synthase (iNOS) prone to release reactive oxygen/nitrogen species (ROS/RNS) inside the resulting phagolysosomes. This allows the biological cargoes carried by the initial phagosomes to be broken down to the molecular level. ${ }^{[2]}$ To ensure a complete digestion of these pathogens the quantities of ROS/RNS present inside phagolysosomes must be sufficient without being excessively high to prevent their leakage and accumulation inside macrophages cytoplasm. ${ }^{[3]}$ This dual requirement suggested the presence of some feedback mechanism operating inside phagolysosomes in order to ensure a ROS/RNS homeostasis. ${ }^{[4]}$. Such hypothesis was recently shown to be coherent with the outcome of long-time (minutes) electrochemical monitoring of ROS/RNS fluxes produced inside phagolysosomes of RAW 264.7 murine macrophages stimulated by Lipopolysaccharides (LPS) and Interferon-Y (IFN-Y) ${ }^{[5]}$ However, to the best of our knowledge, the monitoring of ROS/RNS production inside phagolysosomes with the sub-millisecond time resolution required to establish the very existence of a fast homeostatic regulation could never be achieved before the present work.

Nanoelectrochemical monitoring of intracellular ROS/RNS at the single cell level is a very efficient and quantitative method that has been applied in cancer cells,,$^{[6]}$ macrophages ${ }^{[4,5,7]}$ and neurons. ${ }^{[8]} \mathrm{We}$ recently reported the fabrication of a novel kind of platinized nanowire SiC@C electrodes (Pt-NWEs) and demonstrated their perfect suitability to analyze ROS/RNS inside single phagolysosomes of living macrophages. ${ }^{\left[{ }^{[9]}\right.}$ Phagolysosomes may then randomly collide onto the surface of a Pt-NWE inserted into a macrophage cytoplasm (Figure 1A). This situation is identical to that which operates during vesicle impact electrochemical cytometry (VIEC), a method initially introduced for quantifying neurotransmitters amounts in liposomes and exocytotic vesicles. ${ }^{[10]}$ The high electric field prevailing at 
the electrode surface over nanometric distances is more than enough for electroporating bilipidic membranes. ${ }^{[10]}$ ROS/RNS contained in phagolysosomes may then spill onto the Pt surface through the ensuing pore and be oxidized giving rise to individual amperometric spikes (Figure 1B).

By analogy to the release of neurotransmitters ${ }^{[11,12]}$ it is expected that in the absence of any ROS/RNS homeostasis all recorded spikes display three main features: (i) a small pre-spike feature evidencing the initial opening of the pore followed by (ii) a rapid increase of the current due to the rapid expansion of the pore up to its maximum size, and (iii) finally an exponentially decaying branch (Figure 1D) representing the diffusion-limited emptying of the initial ROS/RNS content. However, only $25 \%$ of the events $(N=1040)$ presented this expected pattern ${ }^{[10,11,12]}$ (Figures $\left.1 \mathrm{D}, 2 \mathrm{C}\right)$. The large majority $(75 \%)$ of spikes displayed the anticipated behavior only up to a given point $\left(t<t_{\exp }\right.$ in Figure 2A, S2A, C) located on their descending branch. The current then significantly deviated from their expected exponential falloff.

In VIEC experiments exocytotic vesicles or liposomes may only release their initial load of electroactive molecules. ${ }^{[10,11,12]}$ However, phagolysosomes are not inert containers. Their membranes are equipped with pre-assembled NOX2 and iNOS enzymes that are likely to be activated to swiftly produce ROS/RNS at least while phagolysosomes keep their functionality after being electroporated. In other words, the unexpected behavior displayed by $75 \%$ of the events could be the first quantitative evidence of the long-time purported homeostatic activity, provided it is established that the current shoulders correspond to ROS/RNS oxidation. This is fully consistent with a recent report by some of us showing that intact phagolysosomes could produce $\mathrm{NO}$ and $\mathrm{O}_{2}{ }^{*-}$ under near steady-state conditions over time durations exceeding tens of minutes. ${ }^{[5]}$

(A)

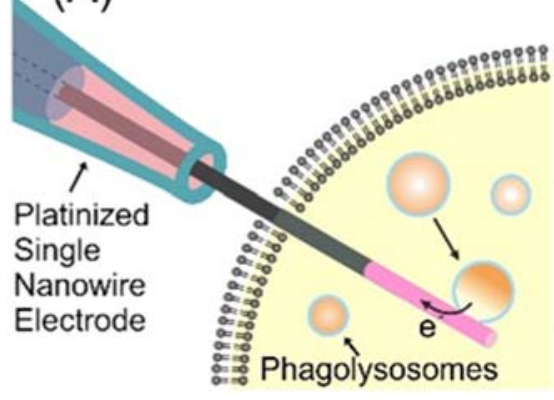

(B)

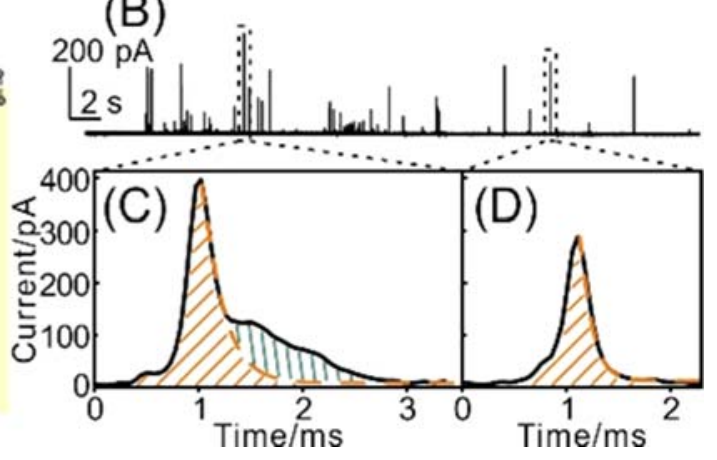

Figure 1. A) Schematic principle of electrochemical detection of ROS/RNS spilling out individual phagolysosomes with a platinized SiC@C nanowire electrode (Pt-NWE) inserted into the cytoplasm of a single macrophage (the pink-colored area at the tip of the NWE symbolizes the black-Pt electroactive coating). B) Typical amperometric trace recorded in a RAW 264.7 murine macrophages. C, D) Magnified resolution of the two representative spikes marked by the dashed rectangles in B. C) spike displaying a clear shoulder superimposed onto its exponential decay branch ( $75 \%$ of events); D) spike displaying a characteristic exponential decay branch ( $25 \%$ of events) as expected in the absence of ROS/RNS homeostatic mechanism. The orange hatched areas in (C, D) represent the contributions of the expected releasing behavior in absence of homeostasis while the green hatched one in (C) represents the superimposed homeostatic contribution (see text and Fig. 2).

However, if the present Pt-NWEs provided an exceptional time resolution in vivo compared to those used in ref. [5], they could not allow a complete chemical characterization of the efflux giving rise to the current shoulders during the short time life of electroporated phagolysosomes ${ }^{[13]}$ Instead, a biochemical approach was implemented by taking advantage that NOX2 and iNOS enzymes may be selectively inhibited by diphenylene-iodonium chloride (DPI) or $\mathrm{N}^{\mathrm{G}}$-monomethyl-L-arginine monoacetate salt (LNMMA) respectively. Macrophages treated with DPI and L-NMMA immediately before the intracellular insertion of a Pt-NWE afforded much fewer and smaller spikes per unit of time. More importantly, their spikes displayed perfect exponential decay branches (Figure 2C). This confirmed that the species responsible for the current shoulders of spikes observed in absence of inhibitors (Figure 2A,B) consisted 
in freshly produced ROS/RNS. Together with the observations reported in ref. [5] this brings strong evidences that phagolysosomes are equipped with efficient means of regulating their intravesicular ROS/RNS content at optimum levels.

For quantitatively investigating the kinetics and intensity of the underlying mechanism we adapted a previous theoretical mode [10i, 11f, 12] developed for rationalizing the kinetics of neurotransmitters release. This allows assessing the ROS/RNS quantities present inside one phagolysosome of released by it in terms of the electrical charge that they deliver upon oxidation at the Pt-NWE surface rather than in moles. The two quantities are proportional through the Faraday law. Herein, the time-dependent quantity of ROS/RNS, ${ }^{[14]} Q_{\text {in }}(\mathrm{t})$, present at time $t$ inside the phagolysosome is then formulated as: ${ }^{[12 \mathrm{c}]}$

$$
\frac{d Q_{\text {in }}(t)}{d t}=-k_{\varrho(t)}^{\text {diff }} Q_{\text {in }}(t)+\frac{d Q_{\text {prod }}(t)}{d t}
$$

where $Q_{\text {prod }}(\mathrm{t})$ is a time-dependent kinetic term accounting for the ROS/RNS production inside the phagolysosome during release; $k_{\mathrm{e}(\mathrm{t})}^{\text {diff }}$ is a pseudo-rate constant featuring the diffusion-controlled release out of the phagolysosome pore of time-dependent radius $\rho(\mathrm{t}){ }^{\left[{ }^{[12 c]}\right.}$ The current, $i(\mathrm{t})$, featuring the ROS/RNS oxidation at the Pt-NWE surface is then: ${ }^{[12 c]}$

$$
i(\mathrm{t})=k_{\mathrm{Q}(\mathrm{t})}^{\mathrm{diff}} Q_{\text {in }}(\mathrm{t})
$$

Introducing Eqn (2) into Eqn (1) and integrating affords Eqn (3):

$$
Q_{\text {prod }}(\mathrm{t})+Q_{\text {in }}(\mathrm{t})=Q_{0}-\int_{0}^{\mathrm{t}} i(\tau) \mathrm{d} \tau
$$

where $Q_{0}$ is the initial ROS/RNS content, i.e., that contained in the phagolysosome just before it is electroporated. ${ }^{[10 \mathrm{~h}, \mathrm{j}] \mathrm{j}} Q_{\text {prod }}^{\infty}$, the limit achieved by $Q_{\text {prod }}(\mathrm{t})$ at the end of the spike represents the total ROS/RNS quantity that has been produced by the phagolysosome while it could maintain its functions after being electroporated (Figure 2B).
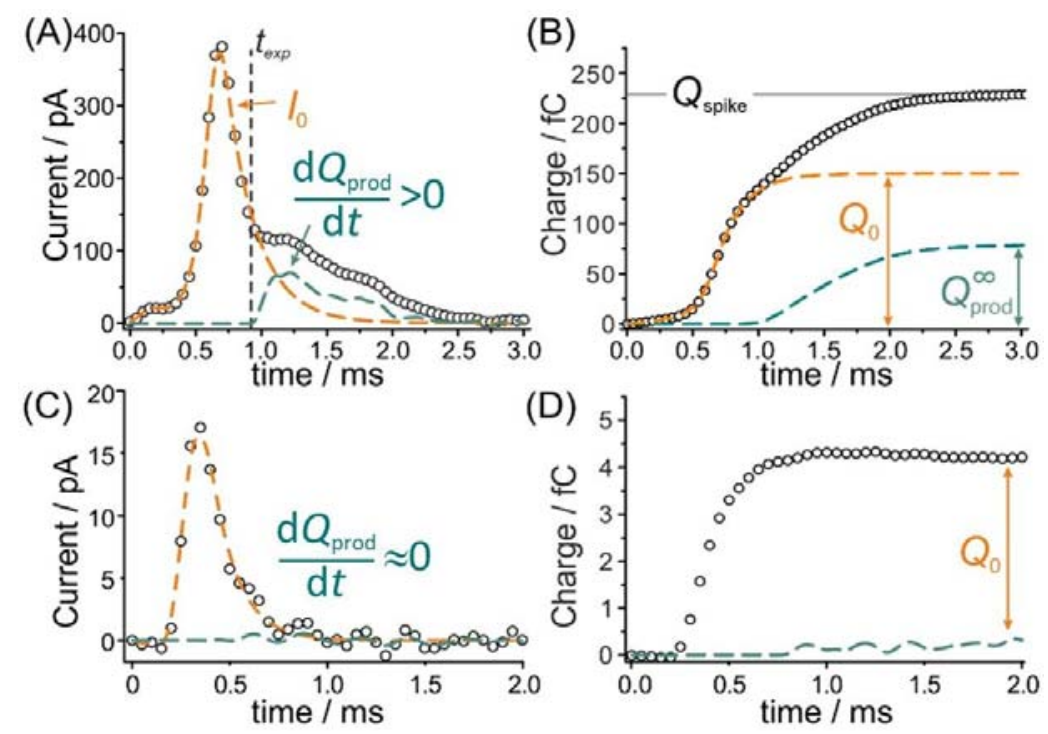

Figure 2. Typical spikes recorded at Pt-NWEs from phagolysosomes (RAW 264.7 murine macrophages) displaying an efficient ROS/RNS homeostasis (A, and two more examples are shown in Figure S2) or after inhibition by DPI and L-NMMA (C), some quantitative data are listed in Table S1. The corresponding charges are displayed in B) for $(A)$, and $D$ ) for (C). In all figures the untreated experimental data are shown in black circles. The orange dashed curves in A) and C) are the reconstructed spikes assuming no homeostasis. The green dashed curves provide the computed rates of ROS/RNS homeostatic production in A) and C) or the corresponding charges in B) and D) respectively. The dashed vertical line in A) marks the instant, texp, at which an efficient production of fresh ROS/RNS began. 
$Q_{0}$ and $k_{\mathrm{e}(\mathrm{t})}^{\text {diff }}$ are intrinsic characteristics of each individual phagolysosome so they are not known a priory. Even so, they can be determined from the theoretical reconstruction of the variations of the spike current when $Q_{\text {prod }}(\mathrm{t})$ is negligible, i.e., for $t<t_{\exp }$ (Figure 2A). Indeed, the theoretical procedure previously developed to account for the treatment of vesicular exocytosis is then readily applicable based on Eqns $(1,2) \cdot{ }^{[12 c]}$ The outcome of this procedure is evidenced by the orange curves reconstructed and extrapolated in Figures 2A,C. (see more detailed description in SI and Figure S1 in $\mathrm{SI}$ for the extracted $k_{\mathrm{e}(\mathrm{t})}^{\text {diff }}$ time-variations for the spikes shown in Figures $\left.2 \mathrm{~A}, \mathrm{C}\right) . Q_{0}$ and $k_{\mathrm{e}(\mathrm{t})}^{\text {diff }}$ being then known, Eqn (3) immediately affords the time variations of $Q_{\text {prod }}(t)$ (Figures 2B,D) whose time derivative gives $\frac{\mathrm{d} Q_{\text {prod }}(\mathrm{t})}{\mathrm{dt}}$ (Figures $\left.2 \mathrm{~A}, \mathrm{C}\right)$. This evidenced that for all $75 \%$ spikes exhibiting shoulder signals the homeostatic process was activated less than $1 \mathrm{~ms}$ after the beginning of release and led to $Q_{\text {prod }}^{\infty}$ values ranging between ca. one third and one half of the initial content $Q_{0}$ (Table S2, Figure 2B). Conversely, for the remaining $25 \%$ spikes $Q_{\text {prod }}(\mathrm{t})$ resulted negligible compared to background current fluctuations (Fig. 1D; compare Figs. 2C, D).

The same procedure was used to compare the homeostatic performances of different phenotypes of RAW 264.7 murine macrophages, i.e., in the resting stage (M0 type) or after their 24h incubation (M1 type) with LPS/IFN-.$^{[4,5,15,16]}$ Both phenotypes exhibited extremely different morphologies and diverse mean intracellular ROS/RNS concentrations as revealed, respectively, by bright-field microscopy and DCFH-DA fluorescent staining (see insets in Figure 3A). ${ }^{[17]}$
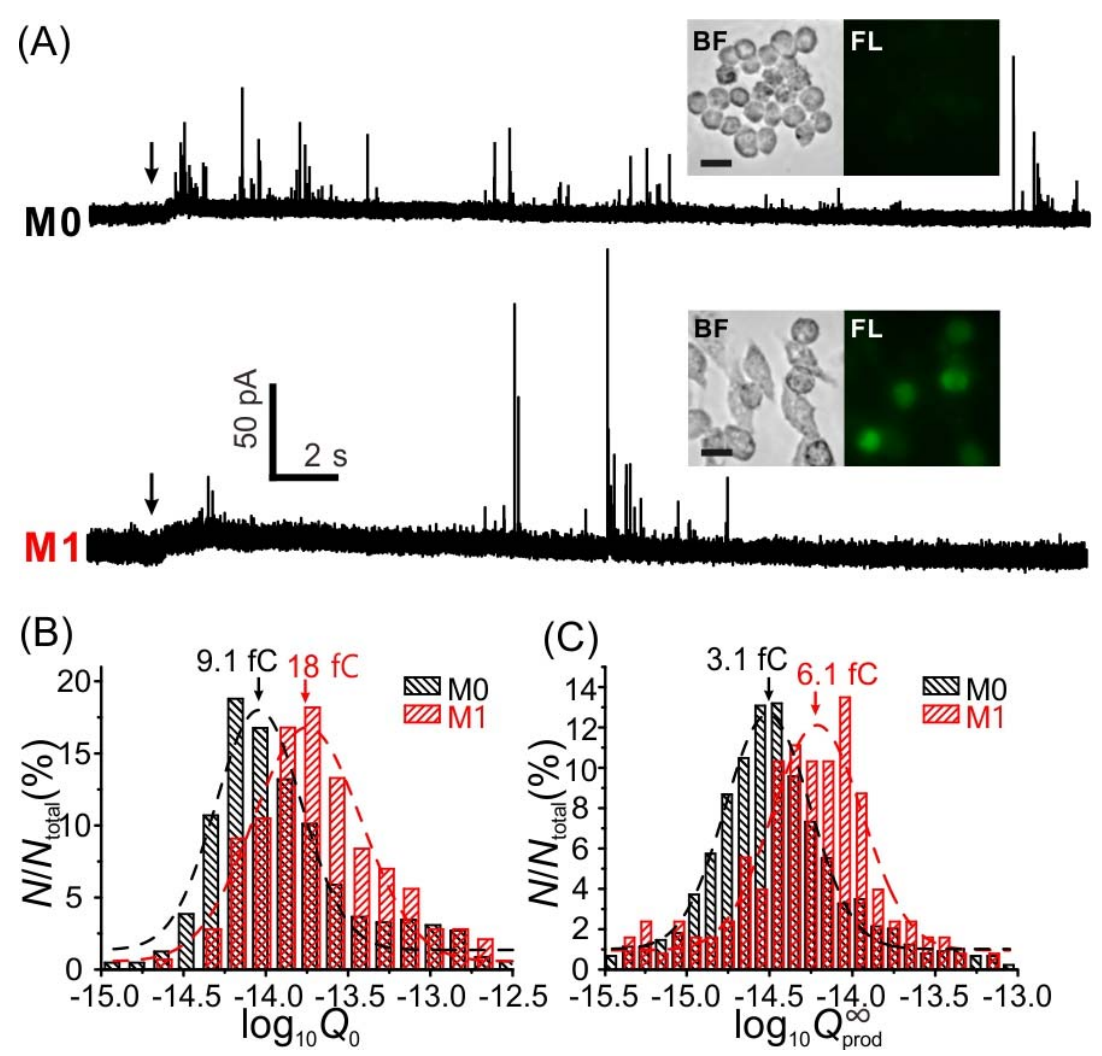

Figure 3. A) Typical amperometric traces recorded inside M0 (upper) or M1 (lower) RAW 264.7 murine macrophages; the same scale bar applies to the two amperometric traces; the arrows indicate the moments of insertion of Pt-NWEs. Insets: optical microphotographs of macrophages (BF: bright field; FL: fluorescence after staining with DCFH-DA); scale bars: $25 \mu \mathrm{m}$. B, C) Distribution of the logarithms of initial, $Q_{0}(\mathrm{~B})$ and freshly produced, $Q_{\text {prod }}^{\infty}(\mathrm{C})$ charges recorded for the M0 (black, 909 spikes from 15 cells) and M1 (red, 127 spikes from 5 cells) phenotypes; arrows indicate the average values (see quantitative details in Table S2). 
Figures 3B, C and Table S2 evidenced that the mean $Q_{0}$ value for $\mathrm{M} 1$ cells (18 $\mathrm{fC}$ ) was nearly double that $(9.1 \mathrm{fC})$ for $\mathrm{M0}$ cells. ${ }^{[18]}$ The same occurred for the homeostatic efficiency as indicated by $Q_{\text {prod }}^{\infty}$ values (6.1 fC for M1 cells versus $3.1 \mathrm{fC}$ for M0 ones). ${ }^{[18]}$ These quantitative differences confirm that LPS/IFN- $\gamma$ activated macrophages contain phagolysosomes generating higher initial level of ROS/RNS $\left(Q_{0}\right)$ and equipped with more efficient biological means for maintaining their homeostasis

$\left(Q_{\text {prod }}^{\infty}\right)$. This may be tentatively ascribed to an up-regulation of enzymes due to the LPS/IFN-Y treatment. ${ }^{[19]}$

In conclusion, this work reported an easily implemented and very efficient experimental and theoretical strategy for quantitative monitoring of ROS/RNS content and production by individual phagolysosomes over sub-millisecond time-scales. This unambiguously unraveled for the first time the existence of a fast ROS/RNS homeostasis and allowed characterizing quantitatively its efficiency under different conditions. We believe that this electrochemical strategy will allow deeper quantitative understanding of phagocytosis.

\section{Acknowledgements}

In China, this work was supported by the National Natural Science Foundation of China (Grants 21725504, 21675121, and 21721005), the University of Xiamen and the State Key Laboratory of Physical Chemistry of Solid Surfaces (PCOSS, University of Xiamen, China) through a Distinguished Scientist Chair awarded to C.A. In France, it was supported in part by PSL University, École Normale Supérieure, CNRS, and Sorbonne University (UMR 8640). Both teams gratefully acknowledge the CNRS Sino-French support (LIA "NanoBioCatEchem").

Keywords: nanoelectrode amperometry $\cdot$ phagolysosomes $\bullet$ ROS/RNS production $\bullet$ homeostasis

\section{References}

[1] R. S. Flannagan, G. Cosío, S. Grinstein, Nat. Rev. Microbiol. 2009, 7, 355-366.

[2] a) F. C. Fang, Nat. Rev. Microbiol. 2004, 2, 820-832; b) I. L. Chapple, J. Clin. Periodontol. 1997, 24, 287-296; c) C. Bogdan, M. Röllinghoff, A. Diefenbach, Curr. Opin. Immunol. 2000, 12, 64-76.

[3] Y. Wang, J. M. Noël, J. Velmurugan, W. Nogala, M. V. Mirkin, C. Lu, M. Guille Collignon, F. Lemaître, C. Amatore, Proc. Natl. Acad. Sci. U. S. A. 2012, 109, 11534-11539. 0.

[4] a) H. Y. Tan, N. Wang, S. Li, M. Hong, X. Wang, Y. Feng, Oxid. Med. Cell. Longevity 2016, 2016, 279509; b) C. F. Nathan, J. B. Hibbs, Curr. Opin. Immunol. 1991, 3, 65-70.

[5] K. Hu, Y. Li, S. A. Rotenberg, C. Amatore, M. V. Mirkin, J. Am. Chem. Soc. 2019, 141, 4564-4568.

[6] a) Y. Li, K. Hu, Y. Yu, S. A. Rotenberg, C. Amatore, M. V. Mirkin, J. Am. Chem. Soc. 2017, 139, 13055-13062; b) R. E. Ozel, G. Bulbul, J. Perez, N. Pourmand, ACS Sens. 2018, 3, 1316-1321; c) H. Zhang, J. Ruan, W. Liu, X. Jiang, T. Du, H. Jiang, P. Alberto, K. E. Gottschalk, X. Wang, Anal. Bioanal. Chem. 2018, 410, 4509-4517; d) X. T. Zheng, W. Hu, H. Wang, H. Yang, W. Zhou, C. M. Li, Biosens. Bioelectron. 2011, 26, 4484-4490; e) R. He, H. Tang, D. Jiang, H. Y. Chen, Anal. Chem. 2016, 88, 2006-2009.

[7] M. Marquitan, J. Clausmeyer, P. Actis, A. L. Córdoba, Y. Korchev, M. D. Mark, S. Herlitze, W. Schuhmann, ChemElectroChem 2016, 3, 2125-2129.

[8] M. Xu, R. Pan, Y. Zhu, D. Jiang, H. Y. Chen, Anal. Chem. 2018, 90, 10117-10121.

[9] X. W. Zhang, Q. F. Qiu, H. Jiang, F. L. Zhang, Y. L. Liu, C. Amatore, W. H. Huang, Angew. Chem. Int. Ed. 2017, 56, 12997-13000; Angew. Chem. 2017, 129, 13177-13180.

[10] a) W. Cheng, R. G. Compton, Angew. Chem. Int. Ed. 2014, 53, 13928-13930; Angew. Chem. 2014, 126, 14148-14150; b) W. Cheng, R. G. Compton, ChemElectroChem 2016, 3, 2017-2020; c) L. Ren, M. D. Pour, S. Majdi, X. Li, P. Malmberg, A. G. Ewing, Angew. Chem. Int. Ed. 2017, 56, 4970- 
4975; Angew. Chem. 2017, 129, 5052-5057; d) X. Li, J. Dunevall, A. G. Ewing, Acc. Chem. Res. 2016, 49, 2347-2354; e) X. Li, S. Majdi, J. Dunevall, H. Fathali, A. G. Ewing, Angew. Chem. Int. Ed. 2015, 54, 11978-11982; Angew. Chem. 2015, 127, 12146-12150; f) N. T. N. Phan, X. Li, A. G. Ewing, Nat. Rev. Chem. 2017, 1, 1-18; g) X. Li, J. Dunevall, L. Ren, A. G. Ewing, Anal. Chem. 2017, 89, 9416-9423; h) X. Li, L. Ren, J. Dunevall, D. Ye, H. S. White, M. A. Edwards, A. G. Ewing, ACS Nano 2018, 12, 3010-3019; i) J. Lovrić, N. Najafinobar, J. Dunevall, S. Majdi, I. Svir, A. Oleinick, C. Amatore, A. G. Ewing, Faraday Discuss. 2016, 193, 65-79; j) see also: B. W. Kim, J. Kim, A. J. Bard, J. Am. Chem. Soc. 2015, 137, 2343-2349.

[11] a) T.J. Schroeder, R. Borges, K. Pihel, C. Amatore, R.M. Wightman. Biophys. J. 1996, 70, 10611068; b) E. V. Mosharov, D. Sulzer, Nat. Methods 2005, 2, 651-658; c) A. Schulte, W. Schuhmann, Angew. Chem. Int. Ed. 2007, 46, 8760-8777; Angew. Chem. 2007, 119, 8914-8933; d) R. Trouillon, M. K. Passarelli, J. Wang, M. E. Kurczy, A. G. Ewing, Anal. Chem. 2013, 85, 522-542; e) Y. T. Li, S. H. Zhang, L. Wang, R. R. Xiao, W. Liu, X. W. Zhang, Z. Zhou, C. Amatore, W. H. Huang, Angew. Chem. Int. Ed. 2014, 53, 12456-12460; Angew. Chem. 2014, 126, 12664-12668; f) Y. T. Li, S. H. Zhang, X. Y. Wang, X. W. Zhang, A. I. Oleinick, I. Svir, C. Amatore, W. H. Huang, Angew. Chem. Int. Ed. 2015, 54, 9313-9318; Angew.Chem. 2015, 127, 9445-9450; g) M. Ganesana, S. T. Lee, Y. Wang, B. J. Venton, Anal. Chem. 2017, 89, 314-341.

[12] a) C. Amatore, A. I. Oleinick, I. Svir, ChemPhysChem 2010, 11, 159-174; b) C. Amatore, A. I. Oleinick, I. Svir, ChemPhysChem 2010, 11, 149-158; c) A. Oleinick, I. Svir, C. Amatore, Proc. R. Soc. A 2017, 473, 20160684.

[13] The platinized nanoelectrodes used in ref. [5] were constructed in such a way that they could penetrate across phagolysosome membranes while ensuring that they immediately resealed tightly around its shaft. This retained the viability of the analyzed phagolysosomes and allowed characterizing and monitoring the productions of $\mathrm{NO}$ and $\mathrm{O}_{2}{ }^{-}$- over several minutes. However, the whole penetration process was too slow to allow any recording of subsecond kinetics. Conversely, if the VIEC-inspired ${ }^{[10]}$ strategy developed in the present work allowed submillisecond time scale resolution it did not allow single phagolysosomes to be monitored during a long time observation presumably due to the lost of functional integrity created by the permanent electoportion. This forbade a complete electrochemical charaterization of the very nature of ROS/RNS efflux. In this respect the two strategies are to be seen as complementary.

[14] C. Amatore, S. Arbault, D. Bruce, P. de Oliveira, M. Erard, M. Vuillaume, Chem. Eur. J., 2001, 7, 4171-4179.

[15] a) D. G. Russell, B. C. Vanderven, S. Glennie, H. Mwandumba, R. S. Heyderman, Nat. Rev. Immunol. 2009, 9, 594-600; b) K. Schroder, P. J. Hertzog, T. Ravasi, D. A. Hume, J. Leukocyte Biol. 2004, 75, 163-189; c) Y. C. Lu, W. C. Yeh, P. S. Ohashi, Cytokine 2008, 42, 145-151.

[16] R. Pan, M. Xu, D. Jiang, J. D. Burgess, H. Y. Chen, Proc. Natl. Acad. Sci. U. S. A. 2016, 113, 11436-11440.

[17] The mean baseline recorded after Pt-NWEs were inserted in the cytoplasm of $M 0(N=9)$ and $M 1$ $(\mathrm{N}=11)$ were twice larger in $\mathrm{M} 1$ cells. These data were obtaind from different series of experiments, and the cell numbers are not the same as listed in Figure 3B, C. Based on the results in refs. [4a, 15] this may suggest a two-fold increased ROS/RNS concentration in LPS/IFN- $\gamma$ activated macrophages. Nonetheless, such data should be taken with due care owing to the comparatively significant background noise.

[18] The "mean" values reported in the text correspond to the mean values of the logarithmic charges values since all quantities ( $I_{\max }, Q_{\text {spike, }} Q_{0}$ and $\mathrm{Q}_{\mathrm{prod}}^{\infty}$ ) obeyed log-normal distributions (Figures 3B, C and S3).

[19] a) M. A. Ellison, G. Thurman, C. M. Gearheart, R. H. Seewald, C. C. Porter, D. R. Ambruso, PLoS One 2015, 10, e0136766; b) U. Boehm, T. Klamp, M. Groot, J. C. Howard, Annu. Rev. Immunol. $1997,15,749-795$. 